\title{
Más allá de las ciencias, los científicos y la gestión de la ciencia y la tecnología Conversación con Hebe Vessuri
}

(1) Patricia Jimena Rivero, ${ }^{1}$ Jorge Andrés Echeverry-Mejía, ${ }^{2}$ Hebe Vessuri ${ }^{3}$

\begin{abstract}
1 Universidad Nacional de Córdoba. Facultad de Ciencias Sociales. CONICET. Centro de Investigaciones y Estudios sobre Cultura y Sociedad CIECS (CONICET y UNC). Socióloga (UAB). Magíster en Estudios Migratorios (UPF). Doctoranda en Estudios Sociales de América Latina (UNC). Correo electrónico: patriciaj.rivero@gmail.com

2 Universidad de Antioquia UdeA. Facultad de Ciencias Sociales y Humanas. Grupo de investigación Redes y Actores Sociales. CONICET. Centro de Investigaciones y Estudios sobre Cultura y Sociedad - CIECS (CONICET y UNC). Licenciado en Comunicaciones (UdeA). Candidato a Magíster en Ciencia, Tecnología y Sociedad (UNQ). Correo electrónico: jorgeandresem@gmail.com
\end{abstract}

3 Instituto Venezolano de Investigaciones Científicas (IVIC). Investigadora emérita. Centro de Investigaciones de Geografía Ambiental (CIGA), Universidad Autónoma de México (UNAM). Investigadora Asociada. Correo electrónico: hvessuri@gmail.com

\section{Introducción}

Estudiar la ciencia y la tecnología implica una mirada amplia que va más allá del análisis de las dinámicas de trabajo de los investigadores y de la configuración de las disciplinas y campos académicos. Significa además indagar sobre las formas en que se está produciendo y utilizando el conocimiento en la actualidad, en contextos locales y globales. Cómo se definen los problemas a investigar, quiénes los definen y a quiénes benefician o perjudican. Esto implica considerar a la investigación como una práctica social similar a otras, con unas características particulares donde intervienen actores y sectores diversos en búsqueda de respuestas y aportes para la comprensión y transformación de las sociedades en que vivimos. Las relaciones entre la ciencia, la tecnología y la sociedad son complejas y requieren de abordajes inter- y transdisciplinarios; existen diferentes tradiciones y contribuciones, particularmente en América Latina, donde se destacan los aportes de la Dra. Hebe Vessuri.

La Dra. Vessuri ha sido investigadora del Consejo Nacional de Investigaciones Científicas y Técnicas (CONICET) en el Instituto Patagónico de Estudios de Humanidades y Ciencias Sociales (IPEHCS) del Centro Nacional Patagónico (CENPAT)-CONICET. También ha sido investigadora visitante del Centro de Investigaciones en Geografía Ambiental (CIGA), de la Universidad Nacional Autónoma de México (UNAM). Se doctoró en Antropología Social por el Institute of Social Anthropology, Universidad de Oxford. Creó la Maestría y luego el Doctorado en Estudios Sociales de la Ciencia y la Tecnología del CENDES, Universidad Central de Venezuela, entre 1977 y 1986. Desde 1987 y hasta 1990 trabajó en el Departamento de Política Científica y Tecnológica de la Universidad Estatal de Campinas. También fue jefa del Centro de Estudios de la Ciencia del Instituto Venezolano de Investigaciones Científicas (IVIC) desde 1991 hasta 2010, y en 1993 fundó el Programa de Posgrado en Estudios Sociales de la Ciencia (Maestría y Doctorado). Actualmente es investigadora emérita de dicho centro.

Es importante resaltar que Vessuri ha contribuido al surgimiento y consolidación del campo de los estudios sociales de la ciencia y la tecnología en América Latina, y es una referente para varias generaciones que se han interesado en las relaciones entre 
ciencia, tecnología y sociedad. Con una extensa participación en el estudio y la gestión de la ciencia internacional, su foco de investigación está en la ciencia en las periferias mundiales y en la interfaz entre la educación superior, la investigación científica y otras formas de conocimiento. Ha llevado a cabo trabajo de campo en México, Argentina, Venezuela, Brasil, Francia y Canadá. Ha obtenido diversos premios. En 2006, el Premio Nacional de Ciencia y Tecnología de Venezuela; en 2014, el Premio Oscar Varsavsky a la trayectoria científica otorgado por la Sociedad Latinoamericana de Estudios Sociales de la Ciencia y la Tecnología (ESOCITE). Recientemente, en 2017, fue reconocida con el John Desmond Bernal Prize de la Sociedad de Estudios Sociales de la Ciencia (Society of Social Studies of Science) a la contribución distinguida, premio que también recibirían en su momento Bruno Latour y Derek De Solla Price.

Es autora y editora de una gran cantidad de libros, artículos, capítulos de libros e informes que ha publicado en varios idiomas y distintas editoriales y revistas de alcance regional y global. Entre sus últimas publicaciones se encuentran: "Latin American science, technology, and society: a historical and reflexive approach", Tapuya 1, 2018 (en coautoría con Pablo Kreimer); "Une transition themématique dans les études STS?”, Revue Anthropologie des Connaissances 11 (2016); 'From science as "development assistance" to "global philanthropy" en The Routledge Handbook on the Political Economy of Science (2017).

A continuación, proponemos un recorrido dividido en tres partes. En la primera, Hebe Vessuri se refiere a la forma en que se ha venido consolidando el campo académico de estudios sociales de la ciencia y la tecnología (CTS), contándonos este proceso de primera mano, dada su participación en iniciativas fundadoras de programas de investigación y posgrado en América Latina. En la segunda parte profundiza en el fenómeno de la movilidad académica y científica en el contexto global, su impacto en la trayectoria de investigadores e instituciones y la manera en que esto se conecta con el aprovechamiento de las capacidades científicas y tecnológicas, dada la importancia de la formación de alto nivel para lograr avances en la generación de conocimientos. Finalmente, en la tercera parte, la Dra. Vessuri se refiere a varios aspectos de la planificación y gestión de la ciencia y la tecnología, considerando el contexto actual argentino y discutiendo sobre la excelencia y la calidad en el sistema científico y tecnológico frente a las posibilidades reales del país y la región, así como los desafíos que plantea el desarrollo de nuestras sociedades.

\section{Primera parte. El campo académico CTS en América Latina}

¿Cómo ves actualmente el desarrollo del campo académico de estudios de ciencia, tecnología y sociedad (CTS) en América Latina? ¿Cómo lo ves configurado? ¿Cómo viene trabajando y qué proyección le ves a futuro?

HV: Todavía es un campo pequeño, aunque está creciendo bien. Es interesante el proceso que se viene dando en las Escuelas Doctorales ESOCITE que hacemos cada dos años de forma rotativa por países de América Latina. Notamos cómo se ha ido haciendo cada vez más en serio, cómo se ha ido profesionalizando el campo. Hay trabajos realmente muy buenos, tesis muy originales, con miradas nuevas y perspectivas innovadoras. Pero eso pasó después de haber llegado a un momento de agotamiento, cuando una tesis tras otra eran sobre el "actor red" de Michel Callon y Bruno Latour; los abordajes se repetían demasiado. Afortunadamente, el campo temático se ha ido renovando, no es que lo del actor red estuviera mal, en su momento fue muy inspirador y renovador, pero han pasado 40 años de eso, entonces, debíamos preguntarnos “¿qué más?”. Ahora se ve un crecimiento, podemos ver que hay un enriquecimiento de los abordajes teóricos, de las miradas, de las aproximaciones metodológicas. Además, ya son varias Escuelas Doctorales en 
las que encontramos algunos jóvenes que están haciendo su doctorado en Europa. Estos jóvenes vienen a la Escuela Doctoral Latinoamericana, lo cual es bueno porque es una manera de "volver a casa". Son chicos nuestros, son latinoamericanos, que se fueron a hacer sus estudios afuera y quieren presentar sus resultados de investigación e interactuar con compañeros latinoamericanos. Eso es muy lindo, porque todo el esfuerzo de hacer $\mathrm{ESOCITE}^{1}$ y las escuelas doctorales de estudios CTS tenía por objeto ayudar a desarrollar esos vínculos de solidaridad, de afecto, ese reconocerse como parte de la misma camada, de la misma cohorte, entre chicos de Colombia, de Argentina, de México, de los distintos países de la región.

Uno de los problemas de nuestros países de América Latina -con baja presencia internacional en esta era de globalización- es estar demasiado aislados, carecer de redes regionales. Estas Escuelas Doctorales y ESOCITE han permitido que la gente con intereses intelectuales compartidos se conozca. De ahí que se hayan establecido lazos profundos de amistad y camaradería, que después influyen en otros ámbitos.

Sin lugar a dudas, la presencia internacional es importante. El campo CTS latinoamericano crece cada vez más. En estos momentos, por ejemplo, ya estamos en el Volumen 1 de la revista Tapuya, que es una revista latinoamericana de Estudios Sociales, Estudios CTS, que se publica en inglés para dar visibilidad internacional a la producción latinoamericana. Y se buscan también autores sobre América Latina de otras regiones del mundo, así como árbitros africanos, hindúes, coreanos, etc., para la revisión de papers latinoamericanos, y viceversa, para que se conozcan mejor. El editor jefe es Leandro Rodríguez Medina, argentino que vive en México, y entre las personas de su comité editorial están Tania Pérez Bustos y Luciano Levin. Los tres se conocieron en la Escuela Doctoral de Caracas. Y así han seguido otras escuelas en Santa Catarina, en Costa Rica; la última fue en Bogotá, en 2017. Se empieza a ver que sí fructifican esas cosas que queríamos encarar con una organización regional para ver si cambiábamos un poco la situación de que los referentes fueran nada más los del primer mundo. Es importante que podamos compartir con el mundo más allá de nuestro país y región, pero como iguales, como diferentes en la igualdad, diversos, plurales. Para ello tenemos que construir escuelas; escuelas en el sentido de que nos podamos reconocer mejor entre todos, fortalecernos en nuestra identidad, conocer cuáles son las dinámicas de nuestros grupos y que podamos tener flujos de intercomunicación más ricos y densos. Poco a poco se está dando, aunque es difícil porque nunca hay dinero. Hay dinero para ir al norte, pero poco o nada para andar por América Latina, entonces es muy difícil.

Vos fuiste la fundadora de la primera Maestría CTS de América Latina. ¿Todavía existe la carrera?

HV: Sí, todavía está. Esa fue la del CENDES -Centro de Estudios del DesarroIlo-, que depende de la Universidad Central de Venezuela, la primera universidad del país. La Comisión Económica para América Latina y el Caribe (CEPAL) tuvo una influencia directa en la creación del CENDES, en la época que siguió a la dictadura de Pérez Jiménez, cuando se empieza a trabajar sobre la nueva institucionalización del Estado. Para la modernización de Venezuela crean una nueva estructura del Estado venezolano, la Oficina Central de Coordinación y Planificación (CORDIPLAN) de la Presidencia de la República. Toda esa estructura de gobierno estaba imbuida de la idea de la planificación, que era muy fuerte en América Latina. El CENDES fue un centro muy importante de la región en ese ámbito; todo el mundo de la CEPAL y Naciones Unidas pasaba por ahí (Vessuri, 2009). En un momento dado, los compañeros venezolanos decidieron armar el área de Ciencia y Tecnología, y justo coincidió con mi llegada como exiliada.
1. La Asociación Latinoamericana de Estudios Sociales de la Ciencia y la Tecnología (ESOCITE) es una organización que tiene por objeto el análisis de las relaciones entre las sociedades y la generación y aplicación de todo tipo conocimiento -incluido el científico y tecnológico-. Desde 1995, ha desarrollado jornadas regionales para discutir los últimos trabajos de ciencia, tecnología y sociedad. Para más información se puede consultar: http://www.esocite.la/ 
2. Oscar Varsavsky fue un importante intelectual latinoamericano reconocido por su cuestionamiento al "cientificismo" y por la búsqueda de la interdisciplinariedad. Una de sus obras principales fue Ciencia, política y cientificismo (1969), en la que propone otro tipo de ciencia, preocupada por las situaciones locales $y$ regionales y no enfocada en los intereses de las élites mundiales.
Llevaba una carta del Consejo Latinoamericano de Ciencias Sociales (CLACSO) para Manuel Sadosky, que estaba exiliado también ahí en el CENDES. Y otras para un par de investigadores que eran dos hermanos, el sociólogo estrella de Venezuela, José Agustín Silva Michelena, coordinador de investigaciones del CENDES, y Héctor Silva, que era economista en la Universal Central. Un día, después de trabajar allí un par de años, José Agustín me dice: “mira Hebe, hemos pensado con Sadosky que sería bueno que armaras el posgrado de Política Científica y Tecnológica”. ¿Cómo? ¿Qué? Y bueno, sí, ¡caray! ¿Cómo vamos a hacer? ¿Qué vamos a investigar? Mis compañeros eran buenos, trabajaban muy bien, eran ingenieros y se interesaban por la tecnología; teníamos un asesor colombiano que trabajaba mucho en políticas públicas. La gente ahí estaba comprometida a hacer un país nuevo. Eso es lo lindo; después de una dictadura, cuando se empieza a pensar y construir las instituciones nuevas para el país; era un momento muy, muy, creativo. Había sido gente muy movilizada en toda esa época de la CEPAL. En ese ambiente, dinámico, creativo, me tocó concebir y dirigir ese primer programa para el que me propuse incluir también la dimensión del análisis social de la ciencia y la historia social de la ciencia.

\section{¿Por ahí pasó Oscar Varsavsky?²}

HV: Sí, Varsavsky estuvo en el CENDES en sus primeros años y volvió más tarde. Y allí dejó su marca. Chávez hizo publicar, entre otros, el libro Ciencia, política y cientificismo. Es decir, hay una edición venezolana que hizo el gobierno, Chávez hablaba del "maestro Varsavsky". En realidad, Varsavsky había sido profesor de su profesor, Jorge Giordani, un colega nuestro en el CENDES, que era economista e hizo el doctorado en la Universidad de Sussex. También daba clase en la Universidad Simón Bolívar. Chávez empezó un posgrado en esa Universidad, y en la bibliografía, Giordani tenía a Varsavsky. ¡Y Chávez había quedado impactado! Más de una vez lo escuché en sus programas de TV de Aló Presidente citar a Varsavsky.

\section{¿Hubo un doctorado en estudios CTS en el CENDES?}

HV: Sí, claro. Y hasta la fecha sigue. Se imparte en el CENDES el Doctorado en Ciencias del Desarrollo, con Mención en Ciencia y Tecnología. Mis primeros alumnos salieron de ahí. Una gran cantidad de gente se formó en esa época. Lo interesante es que de ahí salían para trabajar en el sector público y/o para hacer investigación, carrera de investigación. El doctorado no recuerdo en qué año lo habremos hecho, en el 81, 82, por ahí, salió como programa doctoral formal. La maestría se había hecho antes, habrá sido en el 77, 78. En 1986 me invitan Amílcar Herrera y Renato Dagnino, que estaban en Campinas, para ver si quería ir allá a armar el programa de posgrado de la UNICAMP. Y ahí fui, hice el programa de Campinas, fui la primera directora del Posgrado de Ciencia y Técnica de Campinas.

Por algún tiempo, esos dos fueron los programas doctorales de CTS más importantes en la región. Porque la gente se formaba en el exterior, el doctorado lo hacían en otras partes. Pero pronto en Brasil empezaron otros programas en Sao Paulo, en Río de Janeiro... He tenido la satisfacción -hace poco tiempo, en una visita al Instituto Oswaldo Cruz, en Río de Janeiro- de que el presidente del Instituto, Paulo Gadelha, me invite a comer al salón de investigadores, y empieza a contar cómo en esa época yo era "la" maestra de la gente que estaba trabajando en Brasil en historia de la ciencia. Yo le contesté: "Bueno, en todo caso yo les llevaba a ustedes una semana de ventaja, porque todos estábamos aprendiendo en la misma época"; es decir, quienes estábamos metidos en esto con intereses científicos. Se habla mucho del PLACTED, el Pensamiento Latinoamericano en Ciencia, Tecnología y Desarrollo. Pues los que eran los pensadores del PLACTED eran científicoscientíficos, "científicos duros" que tenían una visión de la ciencia para un país, para la región; querían resolver los problemas y desafíos de esa sociedad. Pero 
después estábamos los que queríamos hacer también un estudio científico, desde las distintas disciplinas: sociología, antropología, historia, etc., sobre esos temas. Yo, por ejemplo, en un momento dado era casi la única que quería trabajar en la temática CTS desde una perspectiva científica, hacer una sociología de la ciencia, o una política de la ciencia o una historia de la ciencia. Es decir, había una cierta distancia entre lo académico y lo político, la militancia política. La diferencia respecto del PLACTED, si había alguna, era esto, además de que éramos una generación más joven. Algunos nos dijeron que éramos reaccionarios, pero lo cierto es que me sentía más científica que militante, quería observar y entender lo que pasaba, y desde la antropología quería hacer estos estudios, comprometidos, pero dándoles el tiempo y la distancia intelectual que me parecían necesarios. Y así fuimos creciendo. Por eso surge el doctorado, porque era gente que quería ir más allá y quería investigar dentro de un espacio académico. Y en eso conté con el apoyo expreso de personas como Amílcar Herrera.

Las escuelas de formación que decís, la Escuela CTS en concreto, ¿creés que ha influenciado en la formulación de políticas científicas en los países de la región? ¿Qué peso creés que han tenido como escuela de pensamiento?

$\mathrm{HV}$ : A veces la gente se encuentra en determinadas circunstancias y se pueden hacer cosas; pero también depende del momento que tomes en cuenta, es diferente si lo ves en el corto, mediano o largo plazo. Por ejemplo, algo que se da como sabido hoy es que el conocimiento científico es una parte muy menor de la política científica. La política científica es política, es negociación, incluye muchas otras cosas. El conocimiento científico entra como algo subalterno, para responder a otras necesidades, otros propósitos, a veces de legitimación en los procesos de negociación de los responsables de la política. Entonces, uno enseña cómo se hace una política, pero si observas a más largo plazo, resurge la importancia del conocimiento, que es lo que transforma el mundo y la manera de entenderlo.

A medida que se profesionaliza el campo, empiezan a emplearse egresados de los programas de maestría como cuadros profesionales de los ONCYTS 3 de la región, aunque no necesariamente haya sido esta una experiencia analizada reflexivamente. Muchas veces, lo que les queda después de finalizar una maestría son unas recetas que, si se aplican ciegamente, seguramente no van a ser muy útiles. Hemos visto cómo evolucionan muchas veces estas políticas, no en el sentido que se quería originalmente, como detonantes para un ejercicio crítico y para seguir en la negociación. Pero la influencia creo que fue recíproca, de la sociedad respecto de demandar egresados de programas de políticas científicas, porque también les daba un caché diferente a los ministerios tener personal con títulos profesionales de alto nivel; les venía muy bien porque aparecían como gente moderna, entonces ahí se engancharon los posgrados. Pero, por otro lado, se perdió un poco el espíritu crítico, los riesgos son esos.

Y a propósito de la consolidación académica del campo CTS y su formación como campo, hace poco Pablo Kreimer publicó un texto sobre la relación tensa entre el campo CTS y las ciencias sociales (Kreimer, 2017) ¿Cómo ves esa relación?

HV: Creo que ese es el pecado de origen, hay una cosa muy de atrás de las ciencias sociales, ellas no querían meterse con las ciencias "duras". Estaban, creo, un poco a la defensiva porque se sentían maltratadas, porque les querían imponer maneras de ver, de teorizar, de ser evaluadas que no respondían a la realidad de las ciencias sociales, a su problemática, a la manera de ser científicos sociales. Y otra, porque pensaban que las ciencias "duras" eran positivistas, no críticas de la manera como lo son las ciencias sociales. Entonces, las ciencias sociales se fueron por otro lado, y en muchos de nuestros países te encontrabas con científicos sociales que estudiaban además de las relaciones sociales y la infraestructura económica o la política;
3. ONCYTS son los Organismos Nacionales de Ciencia y Tecnología que fueron surgiendo en los distintos países de la región latinoamericana. 
también la mitología, leyendas, la magia, la religión, la violencia; en fin, montones de temas muy alejados de las ciencias exactas y naturales. Cuando en Venezuela me pidieron que me encargara de armar un posgrado en política y planificación de la ciencia y la tecnología, pensé: ¿quiénes serán los científicos que tienen más pinta de científicos, que tienen más mentalidad de científicos? Los químicos... ¡Una visión prejuiciada, naturalmente! Pero, en esa sociedad, los químicos se me aparecían como el epítome de la cientificidad, con sus laboratorios, su trabajo experimental, lo húmedo y lo seco, toda esa cuestión. Después, empiezas a ver que hay mucha riqueza en las diferentes prácticas científicas, los médicos que tenían el poder en una comunidad científica nacional; los físicos en otra; y cómo funcionaban unos y otros; qué diferencias hay en cómo se es biólogo, cómo se es bioquímico o ingeniero. El analizar el campo científico resultó ser muy complejo, pues llevaba a romper mitos de la propia ciencia "dura". Entonces, se empezó a explorar esa dimensión. El estudio de las mismas ciencias sociales desde los estudios CTS se hizo más tardíamente; se entró en eso más tarde.

En un capítulo compilatorio del 2014 escribías sobre la vuelta de los estudios del desarrollo (Vessuri, 2014), justo hablábamos del CENDES, de lo que significó en su época. ¿Cómo se estarían trabajando ahora esos estudios de desarrollo? ¿Y cómo se relacionarían actualmente con el trabajo de ciencia y tecnología?

HV:Por un lado, según lo que interpretaba en ese momento -y quizás todavía compro esa idea-, hasta los años sesenta había surgido una sociología académica que se encerró bastante en el ámbito académico dentro de la Universidad: le interesaba construir teoría, criterios de legitimación, etcétera; y en la que se llegó inclusive a decir "nosotros hacemos ciencia social, lo nuestro son problemas sociológicos, no estudiamos problemas sociales, si ustedes quieren trabajar con problemas sociales, váyanse a la calle y sean militantes". Probablemente en el caso particular de Venezuela era una reacción ante situaciones como las que se habían dado con la guerrilla, que surgió en el ámbito de la Universidad, en las residencias estudiantiles de la Universidad Central de Venezuela (UCV), donde estaban las armas y los estudiantes; fue una guerrilla con muchos cuadros académicos. Entonces, la reacción de la academia fue: "si quieres hacer la revolución, eso es un problema social, que se da afuera del recinto universitario; en la Universidad hacemos ciencia, nos dedicamos a una ciencia social, que es una práctica académica”. Y eso fue separándola cada vez más de la dinámica social.

Hubo también una menor valoración de la gente que hacía ciencia social aplicada; un ejemplo es el caso chileno, donde las universidades importantes eran dos: la Universidad de Chile y la Católica, en las que se hacía ciencia académica, tanto social como "dura", pero académica; la ciencia aplicada se hacía en los institutos públicos de investigación. Si eras un investigador en un instituto público de investigación, tu reconocimiento social era bastante menor que si eras profesor de una de esas dos universidades. Entonces, esa desvalorización del investigador que trabajaba en temas del desarrollo porque no era teórico, porque la bibliografía que usaba no estaba en inglés -y por ende, se suponía que no era importante-, llevó a que se convirtieran en dos comunidades contrapuestas, aunque las raíces y su formación fueran las mismas, ya que la formación teórica de los aplicados que se dedicaban a los estudios del desarrollo era la misma que la de los otros. La gente que trabajaba en el desarrollo ni se preocupaba por este asunto, pero estos académicos siempre te miraban "desde arriba".

Además, lo que empezó a verse a medida que pasó el tiempo es también la crisis del estudio disciplinario; darle creciente relevancia a la investigación interdisciplinaria, multidisciplinaria, transdisciplinaria, incluir a otros actores del mundo no científico en el proceso de investigación. Porque incluso en la antropología 
aplicada en los años de auge del desarrollismo, era el investigador quien iba y le decía a la gente qué hacer. Se comprobó repetidamente que eso tampoco funciona necesariamente bien y los resultados fueron, con frecuencia, muy pobres. Entonces se empezó a ver cómo se trabaja con la gente y de ahí el gran descubrimiento de la participación. También se han cometido muchos errores en relación con eso, porque el de la participación es un tema muy difícil y hay límites para la participación del actor no científico en la actividad científica. Pero, obviamente, es un elemento crucial en toda esta historia. Empiezan a surgir diferentes variantes. Yo misma he trabajado un poco con la investigación que llaman "integrada", porque se busca integrar los distintos componentes -actores humanos, no humanos-, los distintos agentes que intervienen.

Otra área de interés reciente es la que tiene que ver con la sostenibilidad, que, además, responde a un discurso que viene de los organismos internacionales, de Naciones Unidas, lo que hace que algunos digan ¿cuál es la trampa? Porque desde el sur uno siempre sospecha cuál será la trampa detrás de todo. Pero también creo que hay una búsqueda genuina de parámetros más duraderos de lo que se hace en los procesos de transformación, para que la vida en la Tierra sea factible más allá del presente. Esta preocupación ayudó a transformar la cosa, a considerar cómo entran otros actores, cómo entran otros saberes, cómo entran otras realidades en el juego; se fue ampliando el ámbito de comprensión de muchas cosas que hasta hace poco quedaban fuera en los estudios.

¿Cómo manejar la tensión entre ciencia y tecnología orientadas hacia la competitividad o hacia la inclusión social?

HV: En nuestro medio se plantean como contrapuestas, aunque creo que no debieran verse así. Un ejemplo muy doloroso es el que se vivió en Venezuela. Allí hubo un total desprecio por la competitividad en aras de una retórica de la inclusión, y no se consiguió ni la inclusión ni la competitividad. Se logró la implosión de la economía, la sociedad y la cultura. Había mucha gente con ganas de participar en el proceso, que decían “¿Cuándo llega el tiempo económico que prometió Chávez?”. Las empresas también son necesarias, por lo menos en la sociedad actual se necesita el apoyo de las empresas para producir. Pero el discurso oficial era: "ese es lucro privado ilegítimo; esa es la rapiña de las multinacionales". Sin embargo, más allá del discurso encendido y vacuo, lo que se dio no fue una política económica "socialista" o "desarrollista" sino un proceso de desindustrialización severo a favor de un sector importador-financiero que se apropió de la renta petrolera en base a fraudes masivos en las importaciones. Competitividad e inclusión social no están necesariamente contrapuestas. Tenemos ejemplos como los de Finlandia, Suecia, inclusive Noruega o Suiza, donde hay empresas competitivas e inclusión social con alta participación y organización sindical en los dos primeros.

\section{¿Cómo ves el rol de los "expertos"?}

$\mathrm{HV}$ : ¿Qué es ser un experto? Hay una literatura abundante sobre el tema. La figura del experto ha estado asociada a la especialización creciente del conocimiento científico, que, más allá de las disciplinas, pasó a concentrarse en silos verticales de especializaciones. Y fue surgiendo un mercado de expertos para dirimir disputas cognitivas, o para establecer criterios de autoridad de conocimiento científico que cada vez más se asoció con el mundo de los negocios, crecientemente ligados a los logos de las instituciones donde obtenían sus títulos y/o donde trabajaban. Lo que se dio en el caso que les menciono fue un proceso de crecimiento de la institucionalidad científica-técnica, con el Consejo Nacional de Ciencia y Tecnología (CONACYT) en Paraguay hace unos 15 años, en Bolivia más o menos también en la misma época, y empezó a surgir la posibilidad del full time dedicado a la investigación y docencia. En Ecuador con Correa también surgió la posibilidad de 
4. Conferencias de consenso. Una conferencia de consenso incluye la opinión del público lego en la toma de decisiones a través del involucramiento público. Lo que se busca con este tipo de conferencias -que reciben diferentes nombres en distintos países- es calificar las actitudes de la gente, en la medida que se les proporciona la información que necesitan hasta que están listos para evaluar una tecnología dada. Generalmente se las considera adecuadas para temas que son socialmente relevantes o que necesitan regulación, para los que el apoyo del público, o la falta de este, es esencial. tener investigación full time en las universidades, de hacer ciencia con la extensa reforma que se hizo en años recientes en ese país. Y así es que empieza a surgir, como institución, también una ciencia social, más allá de las individualidades más o menos talentosas que existieron siempre. Los think tank lo que muchas veces hacen es servir como correa de transmisión de ideologías y modelos importados de otro lugar y penetran la estructura del Estado de maneras sutiles, a través de redes interpersonales. Con la entrada de estos experts y asesores, entra también un nuevo vocabulario, un nuevo lenguaje, una nueva modalidad.

Lo que parece estar pasando ahora es que vienen por el lado de las políticas públicas. Nuestras políticas públicas-y admito que estoy exagerando por una cuestión de énfasis - se parecen en todas partes, son como recetas y provienen del ámbito internacional, penetrando el tejido social, el tejido de gobierno, y de repente te das de cuenta de que te metiste en un lío. Porque no se está respondiendo necesariamente a las condiciones del país. ¿Qué pasa con los diagnósticos? Son diagnósticos hechos muchas veces a la carrera, buscando cualquier fuente para armar un informe y salir del paso. Eso es peligroso.

En Argentina, por ejemplo, parece haber muy poca cultura de evaluación de las políticas. En Europa es una costumbre evaluar, es parte de todo el proceso vinculado a la política. ¿Cómo ves los procesos de evaluación frente a la sociedad? HV: Además de que es parte del desarrollo de una ciudadanía que se ha acostumbrado a evaluar, hay una población más educada donde las consensus conferences 4 han tenido un papel relevante en la sociedad. Nosotros no tenemos eso, recién empiezan a crecer las acciones y movimientos por una "ciencia ciudadana", pero no hay una tradición de evaluación de los programas y de los evaluadores, de todo lo que significa el ejercicio de la política pública. Desafortunadamente, no se encuentra desarrollado el sentido de responsabilidad pública. Desde los estudios CTS tenemos la doble responsabilidad de acercarnos al aparato del Estado formando los cuadros para modernizar ese segmento de la gobernabilidad que tiene que ver con la elaboración de políticas públicas y acercarnos a la sociedad, a la ciudadanía y a los excluidos. Mientras tanto, resulta que la base de las políticas públicas sigue siendo el ejercicio político de la negociación, etcétera. Entonces, el componente técnico y el de conocimiento siguen siendo mínimos, los usas nomás cuando te sirven.

\section{Segunda parte. Movilidad académica y científica}

En Argentina, muchos científicos y científicas se vieron obligados a exiliarse debido a la represión política e ideológica ocurrida en 1966 con Onganía y posteriormente en 1976, con Videla. Y es a partir de estos acontecimientos que se instala en el país la noción de "fuga de cerebros" acuñada en Gran Bretaña. Esta sería la génesis de los estudios de las migraciones calificadas en Argentina. Este esquema acerca de que los talentos de la "periferia" se convierten en beneficio de los países del "centro" -discurso que aún sigue vigente en la política y los medios de comunicación-, ¿es posible resignificarlo y pensar que esos científicos y científicas que se encuentran en el exterior permitirían estimular la creación, difusión y adaptación de nuevos conocimientos para el fortalecimiento de nuestros sistemas de investigación e innovación? ¿Son estas diásporas un potencial para contribuir al desarrollo de nuestra región? ¿O son una amenaza?

HV: Primero, en los tiempos incluidos en la pregunta, dicen que el tema se instala, al menos en Argentina, después del 66 con los militares. En realidad empieza antes, si no me equivoco, en la década del cincuenta. Las estimaciones para el lapso 1950-1970 rondaban el total de 25.000 argentinos calificados viviendo en el exterior, 
aunque no había muy buenas estadísticas, así que probablemente la cifra estaba sobrerrepresentada. Antes de los militares hubo fuga de talentos o brain drain, por cuestiones políticas, pero también financieras o por oportunidades de carrera. Por ejemplo, el caso de Manuel Bemporad en la década del cincuenta. Bemporad era un físico argentino que armó la Escuela de Computación en la UCV, en Caracas. Hubo científicos argentinos que se fueron, ya en la década del cincuenta, a trabajar en distintos lugares del mundo.

Como bien lo plantean, después de la Segunda Guerra mundial empieza a haber todos esos movimientos; los ingleses empiezan a ir a Estados Unidos, los franceses también, en flujos que se consideran de pérdida para los países. Piensen en el caso de Enrico Fermi, el de la bomba atómica, el físico italiano. Su biógrafo comenta que estos científicos se iban de su país ya formados, con lo que para Estados Unidos era pura ganancia. Estados Unidos estaba buscando hacer la bomba atómica para vencer a Alemania, y ahí llega entonces todo un batallón de migrantes científicos europeos para contribuir a la realización del Proyecto Manhattan. Todos eran científicos que se habían formado ya en Europa. Es el caso clásico, típico, que después va a ocurrir en América Latina. En nuestra región el costo resultaba mucho más doloroso, lo mismo que en el sudeste de Asia y China, para las sociedades a las que pertenecían esos científicos. No era solo lo que eso costaba al erario público. Eran capacidades irremplazables que se perdían para países sin capacidades de recambio. También se ha visto la fuga de los inventores para los países pequeños de Centroamérica.

¿Creés que lo de identificar a estas diásporas como una "amenaza" o pérdida para el desarrollo de nuestras naciones no contribuye a pensar al científico o científica como si fuera un stock que tiene que estar físicamente en un determinado lugar? ¿No es posible incorporar al debate otras categorías como "circulación de cerebros" o "intercambio de cerebros", quizás concibiendo que estas personas -aun estando en otros lugares- podrían contribuir a nuestras agendas de investigación en la región? ¿Es posible o definitivamente tendríamos que hablar de brain drain? HV: Bueno, ayer justamente hablábamos en clase sobre la teoría de Caroline Wagner, que dice justamente que lo que tenemos hoy es una circulación de cerebros, en la que se buscan fórmulas de ganar-ganar, porque los individuos tienen mayor libertad de moverse y la lealtad institucional y nacional disminuye en un mundo de redes. No es tanto que se pierde o se gana, precisamente porque ella argumenta: "están afuera mientras el país no está en condiciones de recibirlos", que es lo que pasó durante muchos años en China y en tantos otros países. Un Premio Nobel de Taiwán que conocí en ICSU (Consejo Internacional de las Ciencias por sus siglas en ingles), hacía ya más de 25-30 años que vivía en Estados Unidos, pero era chino. Justamente estábamos saliendo de una reunión en las afueras de París, cuando aparecieron un montón de adolescentes taiwanesas, con sus uniformes con sombreritos que parecían de escuela inglesa; y cuando vieron a este hombre se enloquecieron queriendo sacarse fotos con él porque era "su" científico, "su" Nobel. Ese señor, que había vivido toda su vida profesional fuera de Taiwán, era reconocido en Taiwán como una de sus grandes personalidades del siglo y, entre otras cosas, además de sus méritos científicos propiamente dichos, había recibido a montones de chinos y de otras nacionalidades asiáticas que iban a trabajar con él o con otros colegas en Estados Unidos, abriéndoles las puertas a la ciencia occidental. Era el aporte a su país y su región, desde la distancia.

Ese también ha sido el papel de iniciativas como el Programa Raíces, 5 en que científicos estaban afuera del país porque en Argentina no podían trabajar como querían o simplemente no podían trabajar. Los uruguayos hicieron algo parecido; igual pasó con la diáspora colombiana gestionada de alguna forma por Colciencias
5. El Programa Raíces (Red de Argentinos Investigadores y Científicos en el Exterior) es un programa de vinculación y de retorno del Ministerio de Ciencia, Tecnología e Innovación Productiva de la Argentina, en el marco de la Dirección Nacional de Cooperación e Integración Institucional. Fue sancionado en 2008 como política de Estado. Su propósito es fortalecer las capacidades científicas y tecnológicas por medio del desarrollo de políticas de vinculación con investigadores argentinos residentes en el exterior, así como de acciones destinadas a promover la retención de investigadores en el país y el retorno de aquellos interesados en desarrollar sus actividades en la Argentina. Para mayor información se puede consultar: http://www. raices.mincyt.gov.ar/index.php 
en un momento dado. Ahí se empezó a hablar de ganancia de cerebros vs. pérdida de cerebros, porque estaban disponibles, el país podía usarlos para desarrollar capacidades en lugares especiales en el mundo desarrollado que de otra manera no hubieran podido, y cuando el país pudiera absorberlos regresaban. $Y$ ahora, más recientemente, se habla de circulación. Con la circulación ya estamos en un mundo distinto, el mundo contemporáneo de la ciencia que ya es tecnociencia; los flujos son globales; los mercados materiales y de capitales, y también ahora de seres humanos, son absolutamente abiertos; pero ojo, para quienes tienen altas capacidades, no para el personal no formado, no calificado, pues para ellos hay barreras en todas partes. Por otra parte, se advierte que ninguna de estas cosas funciona exactamente como en la teoría, y sigue habiendo más flujos unilaterales de gente que se va del sur al norte; las agendas se siguen fijando en el Norte; y por eso se habla de explotación de conocimientos y de conocimiento subordinado, de la invisibilidad de la producción de ámbitos periféricos, de la persistencia de grandes asimetrías entre los distintos centros y los contextos débiles.

Los países centrales tienen políticas de atracción bien claras para este propósito... HV: Hay políticas superclaras de atracción que incluyen facilidades para el reconocimiento de títulos, para el otorgamiento del doctorado, para que te quedes ahí después, trabajando. Hay gente que se queda directamente a trabajar en esos países una buena cantidad de años cuando las condiciones en el país de origen no mejoran. Hay momentos en los que curiosamente parece que los que más vuelven de las estadías en el exterior son los menos capacitados y los más capacitados. La franja que se queda más frecuentemente en el país de destino son los del medio, pero aparecen trabajando en puestos subcalificados, no de acuerdo con su nivel de competencia en el mercado de trabajo. Es decir, trabajas por debajo de tu nivel de competencia, pero con todo estás mejor de lo que estarías en tu país de origen, con pocas excepciones.

En México, un investigador de la Universidad Nacional Autónoma de México (UNAM) que está en el Sistema Nacional de Investigadores (SIN) puede cobrar 6000 dólares por mes. Y en ese país he visto a muchos posdocs extranjeros. Y también profesores españoles, holandeses, daneses que no conseguían cargos fijos en Europa, pues en esos países hay demasiada competencia para que el posdoc pueda estabilizarse en un cargo permanente en una institución del medio académico. De esa forma, terminan emigrando a México. México ha sido un buen destino para captación de recursos internacionales en años recientes; Brasil también; Argentina lo ha sido por la oferta de enseñanza gratuita, pero las condiciones en este país para los chicos que vienen de afuera a estudiar y que tienen que trabajar porque no hay becas suficientes hace que se les alargue la carrera demasiado y se vuelve difícil, aunque tienen el acceso, en principio, abierto. El panorama es complicado.

¿Creés que para que haya "excelencia" tiene que haber movilidad internacional en una parte de toda la trayectoria del científico?

HV: Justamente, depende de la definición de excelencia. Si entre los componentes de la excelencia científica está el reconocimiento internacional, entonces es obvio que tiene que haber movilidad internacional en alguna parte de la trayectoria del científico individual. Está ese matemático famosísimo de la India, Srinivasa Ramanujan, totalmente autodidacta, que lo "descubren" unos ingleses y va a trabajar a Cambridge con uno de los líderes de las matemáticas británicas en su momento, Hardy. Este hombre es uno de los grandes genios de las matemáticas, no lo formó nadie, fue de grandecito con su valijita de cartón hasta Cambridge, pero él había reinventado, redescubierto, la teoría de los números, las series y el análisis matemático. ¿Hubiera sido menos excelente si no hubiera salido de la India? Claro que no, sería igualmente un genio. Desde luego que ahí interviene el tema del 
reconocimiento, el componente internacional en esta fase de la tecnociencia, donde los circuitos están tan internacionalizados, se ha hecho mucho más frecuente. Y ayuda a tener contactos, a no tener miedo de publicar en otro idioma, a mandar a las revistas, te da otra flexibilidad.

Cuando juegas solo localmente es más probable que te quedes encerrado, pero cuando saliste... Entonces, cuando juegas internacionalmente, en el extranjero se te despiertan otras habilidades, conoces a otra gente y tienes la posibilidad de estudiar, de formarte en otro contexto, y de trabajar en otro laboratorio, lo cual ayuda y mucho. Ya se ha constituido como parte de la cultura de formación del científico hacer una o varias estancias en el exterior. Pero todo tiene su lado oscuro, porque también ves mucha gente que asimila una forma mimética de ser y hacer del norte trasladando recetas automáticas del exterior a la realidad local, en lugar de aprender a pensar por sí mismos con las herramientas que se esperaba que obtuviera del exterior.

Además, ahora la trayectoria convencional del científico "exitoso" incluye no solo la movilidad internacional, sino la abundancia de becas posdoctorales. La otra cara de la moneda de eso es que la gente en Europa se pasa transitando entre dos y tres posdoctorados, porque no consiguen estabilidad laboral. Es decir que casi estás por jubilarte y todavía no tienes un puesto estable.

\section{En España pasa mucho eso.}

HV: ¡Es terrible! Tengo anécdotas de ingleses y alemanes, personas que eran los mejores de su generación. Un chico, brillante posdoc, va a Cambridge, "oh, qué bueno, ya la hice, estoy en Cambridge", pasa los siguientes cuatro años y ya cuando va a llegar al quinto, su supervisor ha dejado de prestarle atención, hace dos años que lo ignora, porque ahora tiene "carne fresca" que ha llegado para trabajar con él en otros temas, entonces lo que hiciste con él y para él ya pasó, ya te sacó el jugo, y adiós, al descarte. Entonces, ese joven desesperado trata de conseguir otro posdoc en otro laboratorio. Pero él, que era brillantísimo cuando empezó, ya tiene 30 años, en el ínterin ya se casó, tiene un préstamo hipotecario, tiene una familia y, obvio, está desesperado. Eso en el caso de los varones; las mujeres la pasan peor, especialmente cuando son jefas de hogar con niños pequeños. Algunos terminan yéndose a México, o a Brasil, que todavía les permite alguna estabilidad. Pero son también algunas de las caras de la actual práctica científica, a la que también hoy en día llegó la tan mentada “alienación” de la que alguna vez habló Marx.

En uno de nuestros trabajos de campo se observó que se habían dado casos de "retornos temporales" de algunos jóvenes doctores que fueron repatriados por el Programa Raíces, a través de becas de reinserción del CONICET. Pasados los dos años que dura la beca, y ante la imposibilidad de efectivizar su ingreso a la Carrera de Investigador Científico del organismo, tuvieron que volver a reemigrar. La cuestión es que retornaron desde el exterior con familia, mudanza e ilusión y se terminaron quedando sin nada.

HV: Sí, pueden llegar a darse experiencias muy fuertes, muy dolorosas. Es salvaje la cosa, se juega con las expectativas de la gente y el sistema no necesariamente te da un lugar. No está estructurado de manera responsable para pensar las implicaciones de lo que se está haciendo, aunque se afecta a gente, a vidas de familias enteras. Terrible, pero así funciona la cosa en el "mundo feliz" del presente. 


\section{Tercera Parte. La gestión de la ciencia y la tecnología en la Argentina}

La capacidad de investigación de un país es, en buena medida, un asunto de recursos humanos. En este sentido, ¿creés que la forma en que se estructura el sistema de formación de investigadores que tenemos en Argentina es adecuada? ¿Qué aciertos y desaciertos tiene? ¿Qué experiencia/s interesante/s tenemos en América Latina?

Argentina me resulta un país muy raro todavía, que estoy tratando de entender, por eso no puedo decir nada convencida todavía. Hubo un crecimiento fuerte en Argentina, pero curiosamente hay personas que no tienen memoria y creen que la ciencia en el país empezó en el 20040 2005. Lo cierto es que la actividad científica en el país empezó bastante antes. Y la institucionalidad de la ciencia también. Está muy bien el empuje que se le dio después de la última crisis del 2001-2002 al desarrollo científico-tecnológico, ha sido muy significativo y permitió que Argentina comenzara a recuperarse algo de ese subdesarrollo en el que cayó, que es espantoso. Pero todavía tiene unos huecos en los que nos hundimos todos, porque son huecos de formación, de educación, de cultura, muy serios.

En Argentina no sólo hay falencias en la educación superior, sino en todos los niveles de educación; Argentina tiene problemas muy serios en su base educativa. Después de haber sido un país que se distinguió en la región por su liderazgo en materia educativa, hoy en día es un país subdesarrollado, y más subdesarrollado que otros países de América Latina. Sin quitarle mérito a los Kirchner en el empuje reciente que se le dio a la ciencia y la tecnología, hay algunos cambios que fueron estructurales en la década previa, impulsados durante gobiernos que en otros campos fueron muy negativos respecto de lo que significa reconstruir una sociedad democrática, pero con la creación de la agencia y la transformación de la organización política de la ciencia y la tecnología, ayudaron a que la actividad de investigación empezase a funcionar con mejor basamento financiero y visibilidad en el espectro oficial.

También he escuchado que en años recientes se habían empezado a dar muchas becas, y que el tamaño de la población que entraba en la carrera del investigador iba creciendo exponencialmente...

Se afirma que las inyecciones presupuestarias en ciencia y tecnología que hizo el kirchnerismo tenían como propósito cumplir con los objetivos del "Plan Argentina Innovadora 2020". Actualmente se calcula un promedio de tres investigadores por cada 1000 personas económicamente activas, y la proyección que se proponía el Plan era llegar a cinco investigadores por cada 1000, siguiendo los indicadores que tienen actualmente los países centrales.

HV: No lo sé, el ministro puede decir que "hemos entrado en un período de vacas flacas, bajan precios de los productos primarios, tenemos dificultades, está el déficit fiscal, esto y aquello, hay problemas en el Estado, estamos en crisis, tenemos que recortar". Y no hay por qué dudarlo en vista de lo que se ve y escucha en la calle. Pero la sensación que queda, viendo las cosas desde afuera, es que se bajó de golpe, sin anestesia, una ventana de oportunidad, y enseguida, como respuesta a las protestas que naturalmente iban a ocurrir, se empieza a dar becas por aquí, por allá, sin ton ni son, mientras se trata de pasarle el paquete grueso a las universidades, que negocien ellas. En fin, es un tema muy complicado, muy delicado, que no conozco, pero dada la historia de la ciencia y la universidad en Argentina en los últimos 40 o 50 años, me parece que con eventos como este se generan más desconfianzas y resentimientos evitables, lo que hace perder un sentido de orden, de expectativas en la comunidad científica, que parecía por fin enrumbada 
y aparecía como un espacio de optimismo en la Argentina contemporánea, con lo que cuesta crear una cultura de investigación.

Podemos estar de acuerdo o no con la cultura CONICET; uno puede criticar muchas de las reglas de la cultura CONICET, su excesiva rigidez burocrática, etc. Pero el logro de haber instaurado una cultura de investigación en el país es importante y se aprecia solo cuando se pierde. En Argentina se desarrolló una cultura de investigación, sus integrantes empezaron a aceptar las reglas de juego que se establecieron, hay convocatorias, hay expectativas de recuperación de lo perdido y crecimiento a futuro, etc. Además, se mandan señales de cambio que pueden ser peligrosas, pues se corre el riesgo de desvirtuar también el proceso de hacer investigación científico-técnica. Pareciera que ahora todo tiene que ser para la competitividad, por áreas estratégicas, para la innovación. La Argentina 2020... oye, estamos en 2018. Tendríamos que estar haciendo la Argentina 2040 ¿no? ¿Y qué pasó con esta Argentina 2020? ¿Qué ha pasado con los programas? ¿Qué pasó con Pampa Azul y tantas cosas que se propusieron? Hasta hace poco pensaba que podía ser útil convocar a un debate amplio sobre la ciencia, pero la política y la economía nacional no dejan espacio para el diálogo en estos momentos. Aunque creo que podrían salir cosas interesantes, porque en última instancia, la gente, en la investigación científica, está preocupada no solamente por su salario, por su ingreso, sino por el rumbo que va a tomar este país.

A modo de ejemplo acerca de estas cuestiones que implican una mayor reflexión y discusión, tenemos el Programa Raíces, que se convirtió en 2008 en política de Estado. En Internet se puede encontrar dicha ley; es un documento de dos páginas. Lo que resulta extraño es que después de casi una década de implementación de la ley, aún no haya sido reglamentada, sobre todo cuando estamos hablando de una política de Estado, de una bandera nacional. Puede ser peligroso para la salud de nuestro sistema científico y tecnológico, porque entonces viene el gobierno de turno, regula según sus intereses y en un santiamén desaparecen la política de Estado y las garantías de que la política se cumpla. ¿Qué pensás al respecto? HV: Sí. Y no tienes a quién llorarle, no puedes decir nada. Ha sido lo suficientemente importante, ha movido una cantidad de recursos, ha movido una cantidad de gente durante muchos años, ya son muchos años que se ha venido reconstruyendo una capacidad científica. Y fue una iniciativa de los propios científicos, no del Estado, que tardíamente aparece convirtiéndola en política de Estado. Pero ¿cuáles son las implicaciones de eso, más allá del discurso? Yo supongo que la nueva comunidad científica, la nueva capacidad científica debería ayudar a reformular la capacidad universitaria, después de que le sacaron la investigación de su medio. En el tiempo, esa suerte de lobotomía institucional se fue reconstruyendo, con parches por aquí y por allá, como los centros de doble dependencia -que por supuesto aplaudimos que existan, porque muestran que en el país se hace ciencia de calidad-, pero no resuelven el problema central. Se necesitan acciones más profundas e integrales. Aquí en Argentina he visitado algunas instituciones que dan pena por lo que aparece como actividad de investigación, aunque lo que presentan son simulacros tristes y eso probablemente se deba a que quienes están allí no saben cómo hacerlo. A esta altura de la historia...

¿Cómo ves los procesos de planificación estratégica, de gestión de la ciencia y la tecnología y definición de prioridades?

HV: Es útil tener temas estratégicos. Difiero en la metodología de trabajo para llegar a ellos, impulsados siempre por la urgencia. Suelen acabar siendo procesos en petit comités, opacos, donde entre gallos y medianoche se definen las líneas de construcción del futuro. Como cuando, por ejemplo, se pidió a la comunidad del campo que bajaran tres líneas estratégicas para las ciencias sociales, que es un 
6. En el momento de la entrevista existía el Ministerio de Ciencia, Tecnología e Innovación Productiva de la Nación (MINCYT), sin embargo, para el momento de edición de este texto el ministerio fue degradado a Secretaría de Ciencia, Tecnología e Innovación Productiva, dependiente del Ministerio de Educación, Cultura, Ciencia y Tecnología. ámbito inmensamente variado, con montones de líneas de interés... por medio de una consulta administrativa en un lapso reducido.

Tal vez haya que intervenir en el asunto científico desde las bases de la práctica científica, más allá de la estructura CONICET-MINCYT, ${ }^{6}$ a pesar de la escasez de recursos, buscando quebrar el verticalismo o, al menos, flexibilizarlo. Uno de mis intereses ahorita es conocer la Patagonia, la gente que vive y hace proyectos en y sobre su región. En este sentido, encontré distintos grados de frustración de la población en general, y en particular de los científicos que hacen vida en la Patagonia, inclusive investigadores del CONICET. Los escuché decir algo que se pudiera resumir en "estamos acá, deslomándonos en estos lugares difíciles, haciendo patria porque estamos investigando cuestiones relevantes; pero los temas que conciernen al petróleo, por ejemplo, se deciden en Buenos Aires y en La Plata, sin consultarnos ni hacernos participar realmente a los que estamos aquí, viviendo el día a día en el territorio". Por otro lado, algunas instituciones asentadas en esa y en otras regiones tienen con frecuencia falencias serias de capacidades y una selección negativa de las capacidades, cerrándoles la puerta a jóvenes que estarían dispuestos a iniciar o incluso a hacer una carrera de pioneros en la investigación.

En lugar de un círculo virtuoso, se establecen enclaves "duros" de resistencia al conocimiento, desvirtuando el esfuerzo financiero desde el Estado nacional y provincial e impidiendo cualquier despegue en las actividades productivas que cada tanto se ensayan. En varias provincias y regiones del país pasan estas cosas. Entonces, ¿cómo se renegocia todo eso? Y más en un momento de crisis, que pone en evidencia que debe haber un papel más fuerte de las distintas provincias dentro de la negociación económica, social, política, que tiene que ser distinto el procedimiento.

Me asusta comprobar que siguen presentes tantas rémoras del pasado en las formas de negociación del Estado central con las provincias. Recuerdo que hace muchos años estábamos haciendo trabajo de campo en Santiago del Estero, en una visita al norte de la provincia en la cuenca del río Salado, unos lugares muy pobres, donde se había acabado el quebracho y quedaba una erosión muy grande, era un desierto con bolsones de polvo en la carretera de tierra. Un día llega el coordinador del programa de la Fundación Bariloche, para la cual yo estaba haciendo una consultoría antes de irme de Argentina. Esta persona era un científico social reconocido de Buenos Aires en esa época, llegó impecable, en un helicóptero que bajó ahí mismo después que ayudamos a espantar a unas vacas que estaban en lo que era una protopista de aterrizaje. El hombre estaba con unos zapatos de cuero muy lustrados, un impermeable, saco y camisa sport, superelegante en ese polvaderal en que nos encontrábamos. Charló con nosotros tres tonterías y se fue de vuelta, ese era su rol representativo/supervisorio/de guía de la agencia coordinadora en ese momento. Y nosotros allí, en el terreno, nos tuvimos que quedar a dormir en la comisaría del lugar porque era una época muy conflictiva y los trabajadores locales del ferrocarril habían puesto "clavos miguelitos" en la carretera y era peligroso salir llegando ya la noche. Cuarenta años después escucho cuentos similares sobre técnicos, encuestadores, expertos o funcionarios que llegan a los lugares de manera y con estilo semejante. Pareciera que el tiempo no ha corregido nada.

Por último, comentaste hace un momento que no te gusta la palabra y el concepto de "excelencia", de "ciencia de excelencia" ¿Cómo ves este tipo de categorías? HV: Publicamos un trabajo en 2013 con Ana María Cetto y Jean-Claude Guédon (2013); ella es una física mexicana y él un historiador de la ciencia franco-canadiense que se entusiasmó con el tema del acceso abierto hace muchos años. Nuestro artículo apuntaba a defender el concepto de calidad en contra de la noción de 
excelencia. La idea básica es que no debemos tentarnos con el brillo de la excelencia, de las mejores individualidades, sino que, como responsabilidad pública, tenemos que elevar los niveles de calidad de la ciencia, de la educación superior, de la educación secundaria, de la sociedad en su conjunto. La argumentación gira en torno a eso, que en última instancia tiene que ver con la evaluación, y con el acceso abierto, que en teoría abre increíblemente la posibilidad de acceder a la información y al conocimiento. Hacemos una breve historia de cómo en nuestra región evolucionaron en dirección contraria el acceso abierto y las reglas doradas del CONICET, de los ONCYT en general, porque todos hacen lo mismo. Apuntábamos a eso, a la defensa de la calidad más que de la excelencia. En la analogía deportiva, en lugar de tener una medalla de oro olímpica, tener una población sana y físicamente resiliente, gracias a la expansión de buenas prácticas deportivas; en la ciencia, de investigaciones robustas y pertinentes. La excelencia puede quedar como un desafío a futuro, pero mejoremos primero lo que tenemos, porque si no, seguiremos ahondando la brecha. Podemos tener unos pocos supergenios que lleguen a ser noticia de tapa de Nature mientras el país es una lágrima.

\section{Financiamiento}

Esta entrevista fue realizada en Córdoba (Argentina), en mayo de 2018, en el marco del Panel de discusión “Dinámicas y tensiones entre la ciencia, la tecnología y el desarrollo local en Argentina y América Latina", donde participaron como panelistas invitados la Dra. Hebe Vessuri y el Dr. Pablo Kreimer. Tanto el panel como esta entrevista se insertan en el proyecto Ciencia, Tecnología e Innovación para el Desarrollo Inclusivo y Sostenible, radicado en el Centro de Investigaciones y Estudios sobre Cultura y Sociedad - CIECS (CONICET y UNC) y la Facultad de Ciencias Económicas de la Universidad Nacional de Córdoba (FCE-UNC), y financiado por el Ministerio de Ciencia y Tecnología de la Provincia de Córdoba (Argentina).

\section{Agradecimientos}

Queremos destacar la participación activa de Hebe Vessuri en el proceso de intercambio, aclaración y actualización conceptual que tuvo en la versión preliminar de este documento; por este motivo, se consideró pertinente su incorporación como coautora. 


\section{Q Referencias bibliográficas}

》 Kreimer, P. (2017). Los estudios sociales de la ciencia y la tecnología: ¿son parte de las ciencias sociales? Revista Teknokultura, 14, 143-162.

»Vessuri, H. (2009). Los primeros 25 años del CENDES. Cuadernos del CENDES (3ra. Época), 71 (mayo-agosto), 161-168.

"Vessuri, H. (2014). Los límites del conocimiento disciplinario. Nuevas formas de producción del conocimiento científico. En P. Kreimer, H. Vessuri, L. Velho y A. Arellano (Coords.), Perspectivas latinoamericanas en el estudio social de la ciencia, la tecnología y la sociedad (pp. 31-43). México: Siglo XXI.

»Vessuri, H., Guédon, J.-C. y Cetto, A. M. (2013). Excellence or quality? Impact of the current competition regime on science and scientific publishing in Latin America and its implications for development. Current Sociology, 62(5), 647-665. DOI: 10.1177/o011392113512839 